\title{
Quantification and predictors of plasma volume expansion from mannitol treatment
}

\author{
Arabühl, P M ; Ballmer, P E ; Krähenbühl, S ; Krapf, R
}

\begin{abstract}
Objective: To determine the effects of acute hypertonic mannitol infusion on intravascular volume expansion and to identify potential predictors of hypervolemia. Design: Measurements of plasma volume and volume regulatory hormones were performed in healthy volunteers before and over $90 \mathrm{~min}$ after acute infusion of $20 \%$ mannitol solution in a therapeutic dose of $0.5 \mathrm{~g} / \mathrm{kg}$ body weight, equalling an average infusion volume of $180 \mathrm{ml}$. Setting: Clinical research unit in an 800-bed teaching hospital in the eastern part of Switzerland. Participants: Eight normal male volunteers. Measurements and results: Baseline plasma volume was determined by the indocyanine green dye dilution technique. Serial plasma protein measurements were performed after mannitol infusion to calculate intravascular volume changes. Mannitol administration resulted in a plasma expansion that persisted for more than 90 min and peaked at $112 \%$ of the baseline plasma volume $15 \mathrm{~min}$ after infusion. Concomitantly, an increase in systolic blood pressure and a fall in plasma sodium concentration occurred. Pharmacokinetic analyses of mannitol distribution and elimination revealed a close relation between plasma volume expansion and mannitol serum concentrations. While renin activity and aldosterone concentrations were suppressed proportionally to the intravascular volume increase, antidiuretic hormone was increased despite notable volume expansion and hyponatremia. Similarly, a rise in atrial natriuretic peptide was detected. Conclusions: Therapeutic doses of hypertonic mannitol cause substantial plasma volume expansion, resulting in increased blood pressure. Plasma volume expansion is related to mannitol serum concentrations and mannitol clearance determines the time required to restore normovolemia. ADH and ANP are potentially aggravating factors of mannitol-induced hyponatremia
\end{abstract}

DOI: https://doi.org/10.1007/s001340050473

Posted at the Zurich Open Repository and Archive, University of Zurich

ZORA URL: https://doi.org/10.5167/uzh-156574

Journal Article

Published Version

Originally published at:

Arabühl, P M; Ballmer, P E; Krähenbühl, S; Krapf, R (1997). Quantification and predictors of plasma volume expansion from mannitol treatment. Intensive Care Medicine, 23(11):1159-1164.

DOI: https://doi.org/10.1007/s001340050473 


\author{
P.M. Ambühl \\ P.E. Ballmer \\ S. Krähenbühl \\ R.Krapf
}

\section{Quantification and predictors of plasma volume expansion from mannitol treatment}

Received: 27 January 1997

Accepted: 18 August 1997
Abstract Objective: To determine the effects of acute hypertonic mannitol infusion on intravascular volume expansion and to identify potential predictors of hypervolemia. Design: Measurements of plasma volume and volume regulatory hormones were performed in healthy volunteers before and over $90 \mathrm{~min}$ after acute infusion of $20 \%$ mannitol solution in a therapeutic dose of $0.5 \mathrm{~g} / \mathrm{kg}$ body weight, equalling an average infusion volume of $180 \mathrm{ml}$. Setting: Clinical research unit in an 800-bed teaching hospital in the eastern part of Switzerland.

Participants: Eight normal male volunteers.

Measurements and results: Baseline plasma volume was determined by the indocyanine green dye dilution technique. Serial plasma protein measurements were performed after mannitol infusion to calculate intravascular volume changes. Mannitol administration resulted in a plasma expansion that persisted for more than $90 \mathrm{~min}$ and peaked at $112 \%$ of the baseline plasma volume $15 \mathrm{~min}$ after infusion. Concomitantly, an increase in systolic blood pressure and a fall in plasma sodium concentration occurred. Pharmacokinetic analyses of mannitol distribution and elimination revealed a close relation between plasma volume expansion and mannitol serum concentrations. While renin activity and aldosterone concentrations were suppressed proportionally to the intravascular volume increase, antidiuretic hormone was increased despite notable volume expansion and hyponatremia. Similarly, a rise in atrial natriuretic peptide was detected.

Conclusions: Therapeutic doses of hypertonic mannitol cause substantial plasma volume expansion, resulting in increased blood pressure. Plasma volume expansion is related to mannitol serum concentrations and mannitol clearance determines the time required to restore normovolemia. ADH and ANP are potentially aggravating factors of mannitol-induced hyponatremia.

Key words Mannitol - Volume expansion - Hormone response $\cdot$ Pharmacokinetics

\section{Introduction}

Mannitol is frequently employed in the setting of intensive care medicine, especially for the treatment of acute renal failure related to cardiovascular operations or severe traumatic injury, and to control cerebral edema.
However, the administration of mannitol is associated with a number of serious side effects, such as volume overload, hyponatremia, metabolic acidosis, hyperkalemia and acute renal failure $[1,2]$. Since these adverse reactions are probably the consequence of overdosing [3], they might be predictable, and thus preventable, if the 
clinician could base his treatment on experimental data concerning the effects of mannitol. Most of these effects are secondary to the osmotic properties of mannitol in plasma and glomerular filtrate which cause an increase in plasma osmolality, extracellular fluid expansion, inhibition of renal sodium reabsorption and natriuresis [4]. The extent to which mannitol generates volume changes and electrolyte disorders and their pathophysiology are incompletely understood. The literature is scarce on studies that have examined these questions comprehensively. Serum concentrations of volume regulatory hormones such as ANP, vasopressin, renin and aldosterone are affected by acute mannitol admininistration [5-9], but their impact has never been investigated in relation to volume effects, mannitol concentrations and pharmacokinetics.

The aim of the present study was to quantify the short-term intravascular volume effect of a therapeutic mannitol dose in normal men. To define factors that predict the time course of volume expansion and regression mannitol distribution and elimination were calculated from pharmacokinetic analyses. To further characterize the mechanisms that induce hypervolemia and hyponatremia, we determined plasma concentrations of volume regulatory hormones such as atrial natriuretic peptide (ANP), antidiuretic hormone (ADH), plasma aldosterone (PA) and plasma renin activity (PRA) after mannitol administration. Our studies demonstrate that mannitol treatment causes significant alterations in vascular volume status with fluid shifts, accompanied by increased systolic blood pressure, a fall in plasma sodium concentrations and changes in hormone levels regulating water homeostasis. Moreover, we demonstrate that mannitol serum concentration and clearance are the major determinants of its volume effects, whereas increases in ADH and ANP may contribute to the development of hyponatremia following mannitol infusion.

\section{Methods}

Subjects

Experiments were performed in eight male, healthy volunteers $(33 \pm 2$ years old; $74.6 \pm 4.2 \mathrm{~kg}$ body weight; $182 \pm 3 \mathrm{~cm}$ height; $1.90 \pm 0.07 \mathrm{~m}^{2}$ body surface area). Participants were non-smokers, abstained from consuming coffee and alcohol, and confined their physical activity to a minimum the day prior to the experiments. None of the volunteers consumed any drugs. The experiments were carried out in the morning after an overnight fast. Water intake was allowed until the beginning of the study. Urine collection was performed during the $24 \mathrm{~h}$ before the start of the experiment to measure creatinine clearance.
Procedure

Volunteers sat throughout the investigation. Two indwelling catheters were placed, one in each arm, for separate infusions and blood sampling. Baseline parameters, including baseline plasma volume $\left(\mathrm{PV}_{\mathrm{bl}}\right)$, were determined $30 \mathrm{~min}$ later. The infusion of mannitol was started $30 \mathrm{~min}$ after the completion of blood sampling for $\mathrm{PV}_{\mathrm{bl}}$ calculation. Hypertonic mannitol $(0.5 \mathrm{~g} / \mathrm{kg}$ body weight, $20 \%$ solution, corresponding to a volume of $2.5 \mathrm{ml} / \mathrm{kg}$ body weight) was administered intravenously over $10 \mathrm{~min}$. Blood was drawn $15,30,45,60$ and 90 min after initiation of the infusion. The amount of blood drawn at each time point was replaced by infusion of isotonic sodium chloride. Blood pressure was determined in a sitting position at baseline, $15 \mathrm{~min}$ after mannitol administration and at the end of the experiment. Urinary bladders were emptied $30 \mathrm{~min}$ before the infusions were started (to determine electrolytes and osmolality) and at the end of the experiment (to determine creatinine, electrolytes and osmolality).

\section{Blood measurements}

Blood was collected in Vacutainer tubes for the determination of electrolytes, proteins, creatinine and osmolality [heparinized blood], as well as for plasma renin activity (PRA), plasma aldosterone (PA) and antidiuretic hormone (ADH) [EDTA blood], and mannitol [whole serum]. For atrial natriuretic peptide (ANP) measurements blood was drawn manually into plastic syringes, to minimize hemolysis, and transferred to polypropylene tubes containing EDTA, to minimize adsorption on plastic surfaces. All tubes were immediately placed on ice and centrifuged within $15 \mathrm{~min}$ at $3000 \mathrm{~g}$ and $4^{\circ} \mathrm{C}$ for $10 \mathrm{~min}$. Separated plasma for the determination of hormones was stored at $-30^{\circ} \mathrm{C}$ and analyzed within 8 weeks. The mannitol concentration in serum and urine was assessed by capillary gas chromatography after derivatization with hexamethyldisilazan [10]. ANP was measured with a radioimmunoassay (RIA) using an antibody against the human $\alpha$ ANP (Eiken Kogaku, Tokyo). ADH, PRA and PA were determined by RIA. Osmolality was measured with a Fiske osmometer (Vogel OM 801, Giessen, Germany), sodium and potassium with an ion selective electrode (Hitachi 717) calibrated with a flame photometer. Creatinine was determined according to the modified Jaffé reaction [11]. Protein concentrations to calculate plasma volume dilution were determined by the Biuret method. The glomerular filtration rate (GFR) was estimated from creatinine clearance.

\section{Plasma volumes}

Baseline plasma volume ( $\mathrm{PV}$ ) was measured by using the indocyanine green (ICG) dye dilution technique as described elsewhere [12]. ICG was administered in a concentration of $0.25 \mathrm{mg} / \mathrm{kg}$ body weight, and blood samples were drawn $2,4,6,8$ and 10 min after injection of ICG. Following centrifugation, $1.5 \mathrm{ml}$ plasma samples were measured photometrically (Perkin Elmers Lambda II) to calculate baseline plasma volumes. The expanded plasma volume $\left(P V_{\text {exp }}\right)$ after mannitol infusion was calculated by relating the baseline plasma protein concentration $\left(\mathrm{PP}_{\mathrm{bl}}\right)$ to the plasma protein concentration [13] at time points $15,30,45,60$ and 90 min after the start of infusion $\left(\mathrm{PP}_{+\mathrm{n}}\right)$ using the formula $P V$ exp $=$ $\left(\frac{P P b l}{P P+n}\right) \cdot P P b l$ 
Table 1 Summary of physical and chemical parameters determined prior and after administration of mannitol. All values are means \pm SEM. All baseline measurements were performed 30 min prior to administration of mannitol. Serum mannitol, electrolyte and hormone concentrations were determined also at 15 , $30,45,60$ and $90 \mathrm{~min}$ after start of mannitol infusion, and are presented as relative changes from baseline (except for mannitol con- centrations). Blood pressure and urinary parameters are shown in absolute numbers, and were assessed at baseline and $90 \mathrm{~min}$ after start of mannitol infusion (BP also after $15 \mathrm{~min}$ ). Baseline creatinine clearance is based on a 24-h urine collection, which was completed $30 \mathrm{~min}$ before mannitol infusion. Creatinine clearance at $90 \mathrm{~min}$ was determined from blood and urine samples collected at the end of the experiment

\begin{tabular}{|c|c|c|c|c|c|c|c|}
\hline & $\begin{array}{l}\text { Normal range } \\
\text { (if applicable) }\end{array}$ & $\begin{array}{l}\text { Prior to } \\
\text { Mannitol }\end{array}$ & $15^{\prime}$ & $30^{\circ}$ & $45^{\prime}$ & $60^{\prime}$ & $90^{\prime}$ \\
\hline Serum mannitol conc. $(\mathrm{mg} / \mathrm{ml})$ & & 0.0 & $2.6 \pm 0.2$ & $1.85 \pm 0.1$ & $1.64 \pm 0.03$ & $1.45 \pm 0.05$ & $1.16 \pm 0.04$ \\
\hline Blood pressure $(\mathrm{mmHg})$ & & $114.5 \pm 5.2$ & $122.3 \pm 2.8$ & - & $-\quad-2000$ & - & $114.3 \pm 4.5$ \\
\hline Plasma volume (ml) & & $2.815 \pm 185$ & $+341 \pm 50 \dagger$ & $+220 \pm 451 \dagger$ & $+201 \pm 43 \dagger$ & $+186 \pm 49 \pm$ & $+135 \pm 46 t$ \\
\hline Serum $\mathrm{Na}^{+}(\mathrm{mmol} / \mathrm{l})$ & $130-145$ & $140 \pm 1.0$ & $-4.4 \pm 0.6 \dagger$ & $-3.491450 .5 \dagger$ & $-2.4 \pm 1.2 t$ & $-2.6 \pm 0.3 \dagger$ & $-2.0 \pm 1.5 \dagger$ \\
\hline Serum $\mathrm{K}^{+}(\mathrm{mmol} / \mathrm{l})$ & $3.4-4.6$ & $3.6 \pm 0.3$ & +0.05 & +0.0 & +0.1 & +0.15 & +0.1 \\
\hline Serum osmolality (mosmol/kg) & $280-295$ & $287.8 \pm 3.1$ & $+3.25 \pm 1.9$ & $+3.6 \pm 1.5 \ddagger$ & $+4.5 \pm 1.3 \dagger$ & $+3.75 \pm 1.4 \dagger$ & $+2.0 \pm 1.1$ \\
\hline ANP $(\mathrm{pg} / \mathrm{ml})$ & & $117 \pm 28$ & +0.0 & $+13 \pm 4 \S$ & $+17 \pm 58$ & $+14 \pm 7$ & $+12 \pm 8$ \\
\hline $\mathrm{ADH}(\mathrm{pmol} / \mathrm{l})$ & $0-6.7$ & $4.2 \pm 3.4$ & $+0.8 \pm 0.37 \ddagger$ & - & - & $+0.1 \pm 0.55$ & - \\
\hline Renin activity $(\mathrm{pg} / \mathrm{m} / \mathrm{h})$ & $0.8-2.5$ & $1.9 \pm 1.0$ & $-0.56 \pm 0.17 \pm$ & $-0.74 \pm 0.17 \S$ & $-0.55 \pm 0.24$ & $-0.7 \pm 0.2 \S$ & $-0.5 \pm 0.14 \S$ \\
\hline Aldosterone $(\mathrm{pg} / \mathrm{ml})$ & $20-120$ & $95 \pm 49.0$ & $-37 \pm 16$ & $-43 \pm 15 t$ & $-47 \pm 16 \%$ & $-50 \pm 16 \pm$ & $-55 \pm 16 \ddagger$ \\
\hline Urine volume $(\mathrm{ml} / \mathrm{h})$ & & $78 \pm 29$ & - & - $\quad$ r & - $\quad-1$ & - & $287 \pm 21 \dagger$ \\
\hline Creatinine clearance $(\mathrm{ml} / \mathrm{min})$ & & 13291453 & - & _- & _- & _- & $135 \pm 4$ \\
\hline Urinary sodium $(\mathrm{mmol} / \mathrm{l})$ & & $59 \pm 19$ & - & - & - & - & $63 \pm 6$ \\
\hline Urinary potassium $(\mathrm{mmol} / \mathrm{l})$ & & $61 \pm 27$ & - & - & - & - & $28 \pm 5$ \\
\hline Urinary osmolality (mosmol $/ \mathrm{kg}$ ) & & $408 \pm 137$ & - & - & - & - & $540 \pm 46$ \\
\hline
\end{tabular}

$\dagger: p$ at least $<0.01 ; \ddagger: p$ at least $<0.05 ; \S: p$ at least $<0.02$

\section{Pharmacokinetic calculations}

Plasma concentration-time curves were analyzed model-independently. The terminal elimination half-life was calculated from the slope of the terminal elimination phase $(\lambda)$ after semilogarithmic transformation as follows: $t 1 / 2=\frac{\ln 2}{\lambda}$. The total plasma clearance $(\mathrm{CL})$ of administered mannitol was calculated as $C L=\frac{d o s e}{A U C}$, where dose is the mannitol dose infused and AUC the area under the plasma concentration-time curve. AUC was calculated by the trapezoidal rule as follows: $A U C=A U C(0-t)+\frac{C(t)}{\lambda}$, where $\operatorname{AUC}(0-\mathrm{t})$ is the area from time 0 to the last time point when a plasma sample was obtained and $\mathrm{C}(\mathrm{t})$ the plasma concentration at this time point. The volume of distribution at steady state was calculated as: $V s s=$ dose $\cdot \frac{A U M C}{A U C^{2}}$, with AUMC being the area under the first moment concentration-time curve, calculated according to Shumaker [14].

The study protocol was approved by the hospital's Ethic Committee. All volunteers gave written informed consent.

\section{Statistical analyses}

The results are given as means \pm standard error of the mean (SEM). Statistics were performed on absolute values by analysis of variance for repeated measurements (ANOVA). Correlation between the different variables was estimated by regression analysis. Probability values less than 0.05 were considered statistically significant. To account for interindividual variability of baseline concentrations, the results for hormone analyses are also presented as mean percentage change from baseline (in the text and Fig. 1BF).

\section{Results}

A summary of all results is given in table 1 .

\section{Mannitol concentrations and kinetics}

Infusion of mannitol in a therapeutic dose $(20 \%$ solution, $0.5 \mathrm{~g} / \mathrm{kg}$ body weight) over $10 \mathrm{~min}$ resulted in a peak serum concentration of $2.6 \pm 0.25 \mathrm{mg} / \mathrm{ml}$ after $15 \mathrm{~min}$ (Fig.1A). Elimination occurred in a biphasic manner with a slightly slower decrease in serum concentrations after $30 \mathrm{~min}$, resulting in a terminal $t_{1 / 2}$ of $79 \pm 7 \mathrm{~min}$. Mannitol plasma clearance equalled $142 \pm 12 \mathrm{ml} / \mathrm{min}$. The volume of distribution was $15.4 \pm 1.1 \mathrm{l}$, reflecting the penetration of mannitol across the vascular compartment into the interstitium.

Effects on plasma volume, blood pressure and urine excretion

In order to assess the volume effect of $180 \pm 9 \mathrm{ml}$ hypertonic mannitol solution, plasma volumes were determined at baseline before the administration of mannitol by the indocyanine green dye dilution technique $\left(\mathrm{PV}_{\mathrm{bl}}\right.$. Changes in plasma volumes 15, 30, 45, 60 and 90 min after the start of infusion were calculated from baseline values and changes in plasma protein concentrations at the respective time points $\left(\mathrm{Pv}_{\exp +\mathrm{n}}\right)$. Infusion of a therapeutic dose of mannitol resulted in a maximal plasma volume increase of $341 \pm 44 \mathrm{ml}$ or $112.2 \pm 1.5 \%$ of baseline volumes 15 min after the start of infusion $(p<0.001$; 

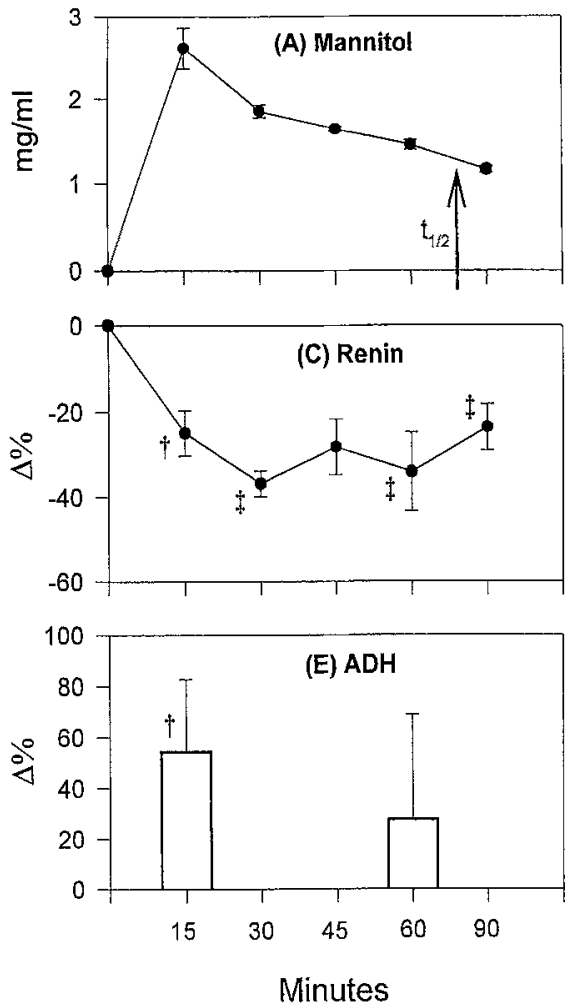

Fig. 1A-F Changes in serum mannitol concentrations (A), plasma volume expansion (B) and endocrine response (PRA (C), PA (D), $\mathrm{ADH}(\mathbf{E})$, and $\mathrm{ANP}(\mathbf{F}))$ after intravenous administration of mannitol: All values are means \pm SEM. Except for mannitol concentrations (absolute values), numbers are presented as mean percent changes from baseline $(=0)$. Time points are indicated as min after start of infusion, which was completed at $10 \mathrm{~min}$. Mannitol concentrations at all time points: $p<0.001$ vs. baseline (all statisti$\mathrm{cal}$ analyses were performed on absolute numbers). Arrow ( $\uparrow$ ) indicates $\mathrm{T}_{1 / 2}$ for mannitol: $p \nmid$ at least $<0.05 ; \ddagger$ : $p$ at least $<0.02$

Fig.1B). Concomitantly, blood pressure rose from $114.5 \pm 5.2$ to $122.3 \pm 2.8 \mathrm{mmHg}$, but returned to baseline values at $90 \mathrm{~min}$ after start of mannitol administration $(114.3 \pm 4.5 \mathrm{mmHg})$. In order to determine the specificity of the blood pressure response to mannitol, isotonic $\mathrm{NaCl}$ was infused to the same volunteers in a separate experiment and in an even larger volume $(420 \pm 25 \mathrm{ml})$ than the resulting plasma expansion from mannitol. Unlike the reactions to mannitol, systolic blood pressure was virtually unchanged by the administration of saline $(116.5 \pm 4.6 \mathrm{mmHg}$ before, and $116.3 \pm 3.8 \mathrm{mmHg} 15 \mathrm{~min}$ after infusion of $\mathrm{NaCl}$ : $p<0.01$ vs increase in systolic blood pressure after mannitol). Mannitol infusion yielded a urinary volume production of $431 \pm 31 \mathrm{ml}$ over $90 \mathrm{~min}$, increasing the urine flow from $78 \pm 29$ to $287 \pm 21 \mathrm{ml} / \mathrm{h}(p<0.0001)$. It is noteworthy that volume expansion lasted for more than $60 \mathrm{~min}$, the time point where the increase in plasma volume equalled the volume of infused mannitol.
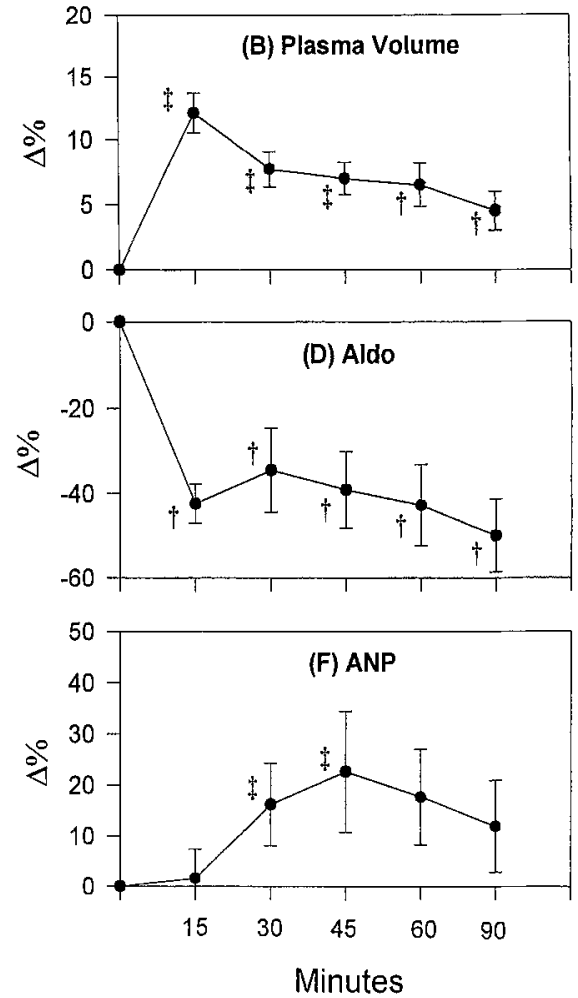

Even after $90 \mathrm{~min}$, when volunteers had excreted more then $400 \mathrm{ml}$ of urine, plasma volumes were still $135 \pm 46 \mathrm{ml}$ above baseline values (Fig. 1A). As for the elimination of mannitol, a biphasic curve was found for regression of the mannitol-associated increase in plasma volume. The initial transcellular volume shift $\left(\mathrm{V}_{\text {shift }}\right)$ induced by mannitol can be estimated from the product of the percent change in serum sodium concentration at $15 \mathrm{~min}$ and the estimated volume of extracellular water $(\mathrm{ECV}=20 \%$ of body weight $)$ : $V_{\text {shift }}=\frac{[\mathrm{NaO}]-[\mathrm{Na15}]}{[\mathrm{NaO}]} \cdot \mathrm{ECV}=\frac{140-135.6}{140} \cdot 0.2 \cdot 751$ $=0.4651$.

\section{Plasma osmolality}

As anticipated, mannitol significantly raised plasma osmolality from $288 \pm 1.1$ to $293 \pm 1.2 \mathrm{mosmol} / \mathrm{kg}$ (Table 1). Whereas time-matched correlations between changes in plasma osmolality following mannitol infusion and intravascular volume expansion missed statistical significance $(r=0.32, p<0.08)$, significant correlations were found when the changes in plasma volume were matched with osmolality changes at the preceding time point. For example, volume expansion determined 30 min after the beginning of mannitol infusion correlated significantly with changes in osmolality measured 15 min earlier $(r=0.82, p<0.05)$. Similarly, the increase 
in plasma volume after 90 min was strongly correlated with the increase in osmolality at $45 \mathrm{~min}$ from infusion $(r=0.94, p<0.01)$. Of interest is the fact that mannitol concentrations were significantly and positively correlated with intravascular volume expansion $(r=0.45$, $p<0.02$ ).

\section{Effect on plasma sodium}

Plasma sodium was significantly lowered by mannitol during the whole experiment, reaching minimal concentrations $15 \mathrm{~min}$ after the start of infusion (Table 1).

\section{Endocrine response}

Plasma renin activity and aldosterone concentrations were lowered significantly after volume expansion due to mannitol (Fig. 1C and D). The mean percentage decreases from baseline for PRA ranged between $25 \pm 5$ $(\mathrm{t}=15 \mathrm{~min})$ and $37 \pm 3(\mathrm{t}=30 \mathrm{~min})$; and for PA between $34 \pm 9(\mathrm{t}=30 \mathrm{~min})$ and $50 \pm 9(\mathrm{t}=90 \mathrm{~min})$.

Despite the marked plasma volume expansion occurring after its administration, mannitol "paradoxically" increased plasma concentrations of $\mathrm{ADH}$ by a mean percentage of $54 \pm 28$ at $15 \min (p<0.05)$ and $28 \pm 41$ at $60 \mathrm{~min}$ from baseline (Fig. 1E). Moreover, the increase in $\mathrm{ADH}$ concentrations after mannitol infusion correlated positively with the early changes in plasma osmolality $(r=0.73, p=0.04)$ but was unrelated to changes in plasma volumes. Finally, ANP concentrations after mannitol infusion were significantly elevated by a maximum mean percentage of $23 \pm 11$ over baseline at $45 \mathrm{~min}(p=0.02 ;$ Fig. $1 \mathrm{~F})$. Unlike $\mathrm{ADH}$, the endocrine response of ANP after mannitol infusion was independent of changes in serum osmolality.

\section{Discussion}

In this study we induced and quantified substantial volume expansion in normal subjects with a pharmacological dose of hypertonic mannitol solution. This was accompanied by an increase in systolic blood pressure and decreased sodium concentrations. We have further elucidated determinants that may account for some of mannitol's adverse effects.

Volume overload is among the most serious side effects resulting from mannitol. The mean infused volume of mannitol $(180 \mathrm{ml})$ in our experiments caused an intravascular volume expansion of up to $341 \mathrm{ml}$ which peaked at $15 \mathrm{~min}$ and persisted for the length of the study (Fig.1B). The total intravascular volume shift $\left(V_{\text {shift }}\right)$ induced by $180 \mathrm{ml}$ of infused mannitol $\left(V_{\text {inf }}\right)$ was $465 \mathrm{ml}$. The calculated volume effect $\left(\mathrm{V}_{\mathrm{eff}}\right)$ of mannitol, expressed as $\mathrm{V}_{\mathrm{eff}}=\frac{V_{\text {shift }}+V_{\text {inf }}}{V_{\text {inf }}}$ was $358 \%$, or about 3 times the administered volume load. The hemodynamic impact of this volume effect is underlined by the observed increase in systolic blood pressure that occurred even in our normal volunteers. This effect coincided with the greatest degree of intravascular volume expansion by mannitol. Such a short-term volume load may significantly compromise the hemodynamic capacity, in particular when projected to patients with impaired cardiac or renal function.

Changes in intravascular volume status were closely related to serum osmolality and serum mannitol concentrations. A tight relationship between the serum mannitol concentration and the plasma volume expansion is further supported by the similar shape of the time curves of the plasma volume and the mannitol serum concentration, with a change in their slope after $30 \mathrm{~min}$. Moreover, our pharmacokinetic data suggest that the regression of plasma expansion is mainly determined by the serum $t_{1 / 2}$ of mannitol, which averaged $79 \mathrm{~min}$. The regression rate of volume expansion between 15 and 90 min after mannitol infusion equals $0.6 \% / \mathrm{min}$. At $79 \mathrm{~min}$ after mannitol infusion, the volume expansion was still $52 \%$ of the initially observed increase, which is close to the $50 \%$ decrease of the mannitol serum concentration at this time point (Fig.1A).

To further elucidate the mechanisms that cause hypervolemia, increased blood pressure and hyponatremia, we investigated the endocrine response to mannitol administration. Whereas the observed decrease in plasma renin activity and plasma aldosterone concentrations is most likely secondary to the mannitol-induced plasma volume expansion, the changes in ADH and ANP require further consideration. It is noteworthy that an increase in ADH after mannitol occurred in the presence of hyponatremia and hypervolemia. This response is attributable to the increase in osmolality and/or plasma mannitol concentrations. As the osmoregulatory mechanism is not equally sensitive to all plasma solutes [9], the resulting stimulatory effect of mannitol and its consecutive rise in serum osmolality was more potent than the inhibitory effects of hyponatremia and plasma volume expansion. The fact that mannitol increases ADH concentration in the face of a fall in plasma sodium concentration could aggravate mannitol-induced hyponatremia clinically, as collecting duct water reabsorption would be expected to be stimulated despite volume expansion and hyponatremia in this setting. Finally, mannitol increased ANP concentrations, most likely by volume expansion, which is the main stimulus for ANP release [15]. This increase in plasma ANP has to be considered with regard to the effects of ANP on urinary electrolyte handling. It is well established that ANP increases renal sodium excretion in a concentration-de- 
pendent manner [16]. Moreover, an additive effect of ANP and mannitol on sodium excretion in dogs has been described when they were infused together [17]. Consequently, the mannitol-induced rise in plasma ANP concentrations may contribute to the natriuresis and hyponatremia resulting from mannitol administration.

What are the clinical implications of our findings? The present data indicate that severe hypervolemia and detectable hyponatremia occur after mannitol administration, even in healthy subjects. It is likely that these effects are even more pronounced in patients with impaired cardiac and renal function prone to develop severe hypervolemia and hyponatremia. However, our pharmakokinetic determinations have shown that mannitol clearance from the serum determines the regression of intravascular volume expansion, and thereby normalization of volume status. Moreover, our experiments suggest that repetitive determinations of serum osmolality are valuable in is predicting the extent of volume expansion from mannitol treatment and detecting patients that warrant special attention. To further evaluate the impact of adapted mannitol treatment, clinical studies based on the present results are suggested in selected clinical settings. From a pathophysiological point of view, the present study proposes a potential role for $\mathrm{ADH}$ and ANP in the development of hypervolemia and hyponatremia resulting from mannitol infusion.

Acknowledgements. We would like to thank Yvonne Ambühl for technical assistance with the study, Dr. Monika Schöpflin (B. Braun Medical Company, St.Gallen, Switzerland) for mannitol determinations and Dr. Joel Melnick for helpful discussions in the preparation of the manuscript.

\section{References}

1. Cottrell JE, Robustelli A, Post K et al. (1977) Furosemide- and mannitol-induced changes in intracranial pressure and serum osmolality and electrolytes. Anesthesiology 47: 28-30

2. Dorman HR, Sondheimer JH, Cadnapaphornchai P (1990) Mannitol-induced acute renal failure. Medicine 69: 153-159

3. Rabetoy GM, Federicks MR, Hostettler CF (1993) Where the kidney is concerned, how much mannitol is too much? Ann Pharmacother 27: 25-28

4. Lang F (1987) Osmotic diuresis. Renal Physiol 10: 160-173

5. Kamoi K, Sato F. Arai O, Ishibashi $M$, Yamaij T (1988) Effects of plasma volume and osmolality on secretion of atrial natriuretic peptide and vasopressin in man. Acta Endocrinol 118: 51-58

6. Yamasaki $Y$, Nishiuchi T, Kojima A, Saito H, Saito S (1988) Effects of an oral water load and intravenous administration of isotonic glucose, hypertonic saline, mannitol and furosemide on the release of atrial natriuretic peptide in men. Acta Endocrinol 119: 269-276
7. Uretsky BF, Verbalis JG, Srinivas M et al. (1990) Control of atrial natriuretic peptide secretion in patients with severe congestive heart failure. J Clin Endocrinol Metab 71: 146-151

8. Kurnik BRC, Lawrence SW, Askenase AD, Kurnik PB (1991) Mannitol stimulates atrial natriuretic peptide release in humans. Am J Kidney Dis 17: 62-68

9. Zerbe RL, Robertson GL (1983) Osmoregulation of thirst and vasopressin secretion in human subjects: effects of various solutes. Am J Physiol 244:E607-E614

10. Knapp DR (1979) Handbook of analytical derivatization reactions. Wiley, New York

11. Bartels H, Cikes M (1969) Chromogens in the creatinine determination of Jaffé. Clin Chim Acta 26: 1-10

12. Bradley EC, Barr JW (1968) Determination of blood volume using indocyanine green (Cardio green) dye. Life Sci 7: 1001-1007
13. Thompson WO, Thompson PK, Dailey ME (1988) The effect of posture upon the composition and volume of the blood in man. J Clin Invest 5: 573 609

14. Shumaker RC (1986) PKCALC: A BASIC interactive computer program for statistical and pharmacokinetic analysis of data. Drug Metab Rev 17: 331-348

15. Lang RE, Thölken H, Ganten D, Luft FC, Ruskoaho H, Unger T (1985) Atrial natriuretic factor: a circulating hormone stimulated by volume loading. Nature 314: 264-266

16. Weder AB, Sekkarie MA, Takiyyuddin M, Schork NJ, Julius S (1987) Antihypertensive and hypotensive effects of atrial natriuretic factor in men. Hypertension 10: 582-589

17. Levy M, Cernacek P (1993) Urinary sodium excretion in chronic caval dogs af ter combined infusions of mannitol and ANP. Am J Physiol 264:F206-211 